\title{
Model Predictive Control of Three Dimensional Spacecraft Relative Motion
}

\author{
Avishai Weiss
}

Ilya Kolmanovsky
Morgan Baldwin

R. Scott Erwin

\begin{abstract}
This paper further develops an approach for spacecraft relative motion control based on the application of linear quadratic Model Predictive Control (MPC) with dynamically reconfigurable constraints. Previous results for maneuvers confined to the orbital plane are extended to three dimensional maneuvers with three dimensional Line-of-Sight (LoS) constraint handling. The MPC controller is designed to prescribe $\Delta v$ impulsive velocity changes rather than piecewise constant thrust profiles. The ability to transition between MPC guidance in the spacecraft rendezvous phase and MPC guidance in the spacecraft docking phase, with requirements, constraints, and sampling rates specific to each phase, is demonstrated. Bandwidth constraints of the spacecraft attitude control system and exhaust plume direction constraints are also addressed. The MPC controller is validated on the full nonlinear model of spacecraft orbital motion and augmented with an Extended Kalman Filter (EKF) to estimate spacecraft states based on relative angles and relative range measurements.
\end{abstract}

\section{INTRODUCTION}

Traditionally, relative motion maneuvers are performed using open-loop maneuver planning and ad hoc error correction. Literature on spacecraft rendezvous control problems is extensive and includes, for instance, [2], [4], [5], [9], and references therein. The XSS-11 [1] is a satellite recently developed by the Air Force Research Laboratory with a focus on demonstrating such relative motion capabilities.

The robustness, fuel efficiency, speed, safety, and reliability of spacecraft relative motion maneuvers can be improved through the application of feedback control algorithms. With this motivation, in [10], [11] and [12] we developed an approach to perform relative motion maneuvers based on the application of linear quadratic Model Predictive Control (MPC) and dynamically reconfigurable linear constraints. The MPC controller uses a linearized relative motion model and linear constraints that are generated online in order to compute the optimal control sequence over a finite horizon; it then applies the first element of this sequence to the spacecraft and repeats the process at the next sampling instant based on the measurements of the spacecraft state. For maneuvers confined to the orbital plane, we demonstrated in [10], [11] and [12] the capability of the spacecraft, controlled with MPC, to approach both a non-rotating and rotating/tumbling platform, while avoiding a piece of debris

This work was supported by ASEE through the Air Force Summer Faculty Fellow Program (SFFP).

Avishai Weiss and Ilya Kolmanovsky are with the Department of Aerospace Engineering, The University of Michigan, Ann Arbor, Michigan. weiss.avishai@gmail.com, ilya@umich.edu

Morgan Baldwin and R. Scott Erwin are with the Space Vehicle Directorate, Air Force Research Laboratory, Albuquerque, New Mexico. afrl.rvsvakirtland.af.mil along the spacecraft's path. In addition, we demonstrated robustness to unmeasured disturbances through the mechanism of systematic feedback corrections with MPC. We have also shown a direct connection between weights in the MPC cost function and fuel consumption and time-to-dock maneuver attributes. Finally, the computational implementation of the linear quadratic MPC approach with dynamically reconfigurable constraints reduces to an on-line solution of a quadratic programming problem, which is computationally feasible on-board a spacecraft. Furthermore, if the spacecraft approaches a non-rotating platform with a known Line of Sight (LoS) cone orientation, an explicit MPC approach that does not require on-board optimization can be used and is based on storing an offline pre-computed MPC law in the form of a piecewise affine control function (i.e., in the form of look-up tables and if-then-else conditions).

In this paper, we extend the approach of [10], [11], [12] in several ways. These include the treatment of three dimensional relative motion maneuvers with three dimensional Line of Sight (LoS) cone constraints, the use of MPC controller to prescribe $\Delta v$ impulsive velocity changes rather than piecewise constant thrust profiles, and the demonstration of the ability to transition between MPC guidance in the spacecraft rendezvous phase and MPC guidance in the spacecraft docking phase, with requirements, constraints, and sampling rates specific to each phase. To avoid the need for a long MPC control horizon or long sampling periods in the rendezvous phase, we employ a referencegovernor like approach, where the desired equilibrium setpoint is replaced by a virtual set-point that is, along with the control sequence, determined through the solution of the Quadratic Programming (QP) problem. In addition, we demonstrate that bandwidth constraints on the spacecraft attitude control system and exhaust plume direction constraints can be successfully handled by introducing an appropriate penalty term in the cost function and a relative-distance dependent constraint on the thrust vector, respectively. We validate our MPC controller on the full nonlinear model of spacecraft orbital motion and we demonstrate that the MPC feedback can be implemented based only on measurements of relative angles and relative range. To accomplish the latter, we augment an Extended Kalman Filter (EKF) to the MPC controller. We present simulation results that are based on a set of spacecraft parameters representative of a small spacecraft with limited thrust capability.

Several other variants of the Model Predictive Control framework, different from our work, have been proposed in the literature. The approach employed by [13] and [2] uses a 
variable length horizon and requires the solution of a mixedinteger linear program at every control step. The "rubber band" MPC approach was proposed in [6]. An application of MPC to spacecraft navigation in proximity of a space station is considered in [16], where an unconstrained MPC is proposed for guidance to the neighborhood of the space station, while the LoS between the station and the spacecraft sensors is maintained by a constrained spacecraft attitude controller, and a control allocation scheme to operate the thrusters. In a similar context, a receding horizon controller that uses the solutions of non-convex quadratically constrained quadratic programs has been proposed in [7] for passively safe proximity operations, where a statistical model of the uncertainty is used for improving robustness with respect to position uncertainty. The approach used in this paper only requires that a conventional quadratic program be solved numerically. The details of our MPC approach are also different from a recent paper [15], specifically, our approach is based on a QP (versus LP in [15]), uses a reference governor style formulation of MPC cost for the rendezvous phase, explicitly handles LoS cone constraints, and accounts for the capability of the attitude control system by an attitude penalty in the cost. On the other hand, our paper does not treat the passive safety requirements or the elliptic orbit case.

\section{MODEL}

In traditional relative motion problems, an approaching spacecraft is maneuvered close to a target spacecraft in a nominal orbit. The target spacecraft is assumed to be at the origin of Hill's frame. The equations of motion for an approaching spacecraft around a target spacecraft depend nonlinearly on the orbital radius. For nominal circular orbits (assumption in this work), the linearized equations of relative motion reduce are the well known linear time-invariant Clohessy-Wiltshire-Hill (CWH) equations [4].

\section{A. Nonlinear equations of motion}

The relative position vector of the approaching spacecraft with respect to the target spacecraft is expressed as

$$
\vec{r}=x \hat{\imath}+y \hat{\jmath}+z \hat{k},
$$

where $x, y$ and $z$ are the components of the position vector of the approaching spacecraft relative to the target spacecraft. The position vector of the approaching spacecraft with respect to the center of the Earth is thus given by $\vec{R}=\vec{R}_{0}+\vec{r}=\left(R_{0}+x\right) \hat{\imath}+y \hat{\jmath}+z \hat{k}$. The nonlinear equations of motion for the approaching spacecraft can be expressed in vector form as

$$
\ddot{\vec{R}}=-\mu \frac{\vec{R}}{R^{3}}+\frac{1}{m_{c}} \vec{F},
$$

where $\vec{F}$ reflects the vector of forces applied to the approaching spacecraft, $R=|\vec{R}|, m_{c}$ is the mass of the spacecraft, and $\mu$ is the gravitational constant. In the simulations in this paper, we consider a set of parameters representative of a small autonomous spacecraft. The spacecraft is in a nominal circular orbit with an altitude of $850 \mathrm{~km}$ above the Earth.

\section{B. Linearized CWH equations}

For $\delta r<<R$, the linearized CWH equations [4] approximate the relative motion of the approaching spacecraft as

$$
\begin{aligned}
\ddot{x}-3 n^{2} x-2 n \dot{y} & =\frac{F_{x}}{m_{c}}, \\
\ddot{y}+2 n \dot{x} & =\frac{F_{y}}{m_{c}}, \\
\ddot{z}+n^{2} z & =\frac{F_{z}}{m_{c}},
\end{aligned}
$$

where $F_{x}, F_{y}, F_{z}$ are components of the external force vector (excluding gravity) acting on the spacecraft. In these equations, $n=\sqrt{\frac{\mu}{R_{0}^{3}}}$, where $R_{0}$ is the nominal orbital radius.

\section{Discrete-time prediction model}

We convert the model (2) to discrete-time to be used as a prediction model in MPC. The effects of thrust are modeled as an impulsive change in the relative velocity of the approaching spacecraft, denoted by $\Delta v$. Assuming the sampling period, $\Delta T$, the discrete-time model has the following form

$$
\begin{gathered}
X_{k+1}=A\left(X_{k}+\bar{B} \Delta v_{k}\right) \\
=A X_{k}+B U_{k},
\end{gathered}
$$

where $X=[x, y, z, \dot{x}, \dot{y}, \dot{z}]^{\mathrm{T}}$ denotes the full state, $U=$ $\Delta v$ is the control, $A$ is the discretized matrix from $\mathrm{CWH}$ equations, $B=A \bar{B}$, and the constant matrix $\bar{B}$ maps the velocity change $\Delta v$ to the full state change.

\section{Constraints}

Rendezvous and docking maneuvers must conform to various constraints [11], [12], [10] including:

- Thrust magnitude and direction limits (so that the spacecraft does not fire its thrusters into the target). The thrust capability limits the velocity change to $10 \mathrm{~m} / \mathrm{sec}$ in each direction.

- Sensor constraints, such as the need for the approaching spacecraft to remain within a Line of Sight (LoS) cone from the docking port located on the target spacecraft [13]. The LoS cone constraints in three dimensions are quadratic, and we handle them through an appropriate inner approximation by polyhedral constraints, $A_{\text {cone }} X \leq b_{\text {cone }}$

- Soft-docking constraints [11], [12], [10] on the terminal velocity of the spacecraft to match the velocity of the docking port,

- Attitude controller capability induced constraints for rate of thrust vector realignment,

- Overshoot constraints, so that the spacecraft does not crash into the target.

\section{E. Discussion}

Due to the multitude of state and control constraints and due to requirements to reduce fuel consumption during maneuvers, Model Predictive Control (MPC) represents an attractive framework for control of spacecraft rendezvous and docking. Typical spacecraft rendezvous maneuvers of 
$10 \mathrm{~km}$ can take several orbits to complete. In comparison, our previous work [11], [12], [10] considered larger levels of thrust, smaller mass and shorter maneuver distances and durations. A special formulation of the MPC optimization problem is necessary to avoid a long control horizon and avoid solving a highly complex optimization problem online. Longer sampling periods are also introduced in the rendezvous phase, when the approaching spacecraft is further away from the target, versus the docking phase, when the approaching spacecraft is closer to the target.

\section{Model Predictive Controller}

An MPC controller generates control actions by applying a moving horizon optimization to a system model subject to pointwise-in-time state and control constraints. The control is periodically recomputed with the current state estimate as an initial condition, which ensures robustness to uncertainties and disturbances.

\section{A. MPC Problem Formulation}

If we use linearized equations of motion, linear equality and inequality constraints, and quadratic costs on the states and control actions, then we can formulate the MPC problem as a quadratic programming $(\mathrm{QP})$ problem. This $\mathrm{QP}$ can be solved in real time using code-generated custom solvers [8].

Based on practical considerations, we separate the problem into distinct rendezvous and docking phases.

In the rendezvous phase, the spacecraft is a significant distance away from the target. We use a sampling period of 120 sec and we enforce a velocity change magnitude constraint. Since the thrust of the spacecraft is limited, to avoid the need for a long control horizon we use a reference governor like approach. In this approach, the desired equilibrium set-point is dynamically modified. Instead of locating the set-point at the origin, we let the QP find forced equilibria between the approaching spacecraft and the target. The rendezvous phase QP is specified in (4), assuming $k=0$ is the current time:

$$
\begin{array}{ll}
\operatorname{minimize} & \sum_{k=1}^{\mathrm{N}-1}\left(X_{k}-X_{\mathrm{s}}\right)^{T} Q\left(X_{k}-X_{\mathrm{s}}\right) \\
& +\sum_{k=1}^{\mathrm{N}-1}\left(U_{k}-U_{\mathrm{s}}\right)^{T} R\left(U_{k}-U_{\mathrm{s}}\right) \\
& \quad+\left(X_{\mathrm{N}}-X_{\mathrm{s}}\right)^{T} Q^{\text {final }}\left(X_{\mathrm{N}}-X_{\mathrm{s}}\right)+X_{\mathrm{s}}^{T} P X_{\mathrm{s}} \\
\text { subject to } & X_{k+1}=A X_{k}+B U_{k} \\
& X_{\mathrm{s}}=A X_{\mathrm{s}}+B U_{\mathrm{s}} \\
& \left|U_{k}\right|_{\infty} \leq u_{\mathrm{max}} \\
& X_{k}, U_{k}, X_{\mathrm{s}}, U_{\mathrm{s}}
\end{array}
$$

When the approaching spacecraft enters a specified box around the target, we switch to the docking phase. During this phase we remove the reference governor type action as it is no longer necessary; the origin, that is the true desired equilibrium can be reached over the optimization horizon under the problem constraints. We reduce the sampling period to $20 \mathrm{sec}$ to facilitate faster control updates. We impose an LoS cone constraint and we treat it as soft via a polyhedral cost penalty. We also impose a thrust direction constraint and an in-track target overshoot constraint. Additionally, we approximately penalize the rotation the approaching spacecraft must perform between thrust impulses by imposing a cost on change in control policy. The docking phase QP, assuming constraints are configured for an along-the-track, V-bar approach, is given in (5):

$$
\begin{aligned}
\operatorname{minimize} & \sum_{k=1}^{\mathrm{N}-1} X_{k}^{\mathrm{T}} Q X_{k}+U_{k}^{\mathrm{T}} R U_{k}+X_{\mathrm{N}}^{\mathrm{T}} Q^{\mathrm{final}} X_{\mathrm{N}} \\
& +\sum_{k=1}^{\mathrm{N}} \lambda \mathbf{1}^{\mathrm{T}}\left(A_{\text {cone }} X_{k}-b_{\text {cone }}\right)_{+} \\
& +\sum_{k=0}^{\mathrm{N}}\left(U_{k}-U_{k-1}\right)^{\mathrm{T}} R^{\text {attitude }}\left(U_{k}-U_{k-1}\right)
\end{aligned}
$$

subject to $X_{k+1}=A X_{k}+B U_{k}$

$$
\begin{aligned}
& \left|U_{k}\right|_{\infty} \leq u_{\max } \\
& U_{k, 2} \leq \mu e^{-\beta k} \\
& x_{k, 2} \geq 0
\end{aligned}
$$

where $U_{k, 2}=\Delta v_{y}$ and $X_{k, 2}=\Delta y$ denote the vector components along the orbital track direction. To improve the response, we have found it necessary to further subdivide the docking phase into two sub-phases, when the spacecraft is more than $100 \mathrm{~m}$ away and when it is less than $100 \mathrm{~m}$ away. We used $R^{\text {attitude }}=2 \times 10^{6} \cdot I, \beta=5, \mu=10^{-2}$ if relative distance is greater than $100 \mathrm{~m}$ and we used $R^{\text {attitude }}=10^{7} \cdot I$, $\beta=1, \mu=10^{-4}$ if relative distance is less than $100 \mathrm{~m}$.

The effects of including the attitude control penalty with $R^{\text {attitude }}>0$ are transparent in Figure 1. The angle between two consecutive velocity changes tends to be smaller with the penalty included, reducing the effort of the attitude control system to change the orientation before the next thrust event.

\section{B. Robustness of $M P C$}

Figure 2 illustrates the robustness of Model Predictive Control (MPC) feedback versus open-loop solutions. Here a $100 \mathrm{~km}$ in-track MPC maneuver that is successfully completed in a linear simulation is simulated open-loop using the nonlinear equations of motion (1) and compared to an MPC solution where the feedback is based on the true state, given by the nonlinear model. Due to differences between the linearized and nonlinear equations of motion, the openloop maneuver does not result in a successful rendezvous with the target. The maneuver that uses systematic feedback corrections based on the true state is successfully completed and the trajectory is very similar to the one using a linear simulation.

\section{Output Feedback MPC Implementation}

The above MPC controller can be implemented using output measurements only. We assume that the relative range and the relative angles are measured. The measurement 


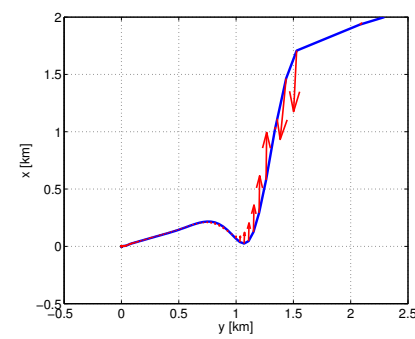

(a)

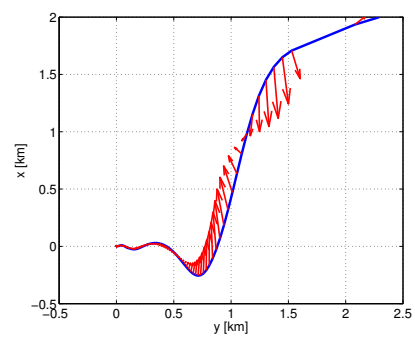

(b)

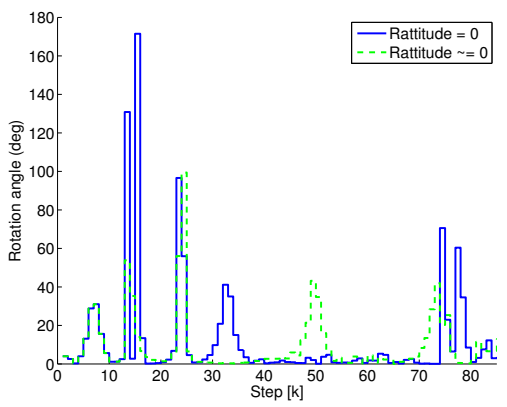

(c)

Fig. 1: Spacecraft trajectory on $x-y$ plane. Arrows indicate the direction and scaled magnitude of $\Delta v$ 's induced by thrusting: (a) With $R^{\text {attitude }}>0$; (b) With $R^{\text {attitude }} \neq 0$. (c) Comparison of the angles between two consecutive velocity changes for the cases (a) and (b).

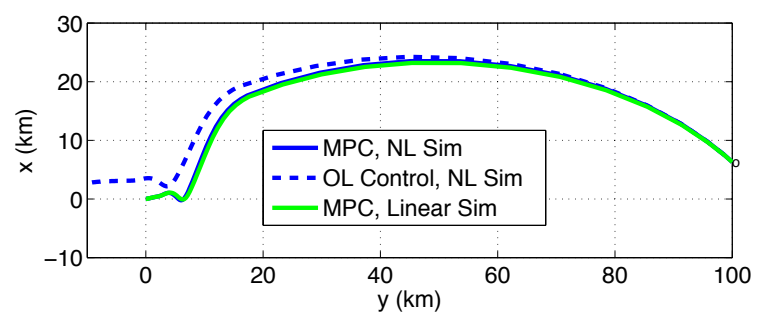

Fig. 2: Spacecraft trajectories on $x-y$ plane for MPC based on a linear simulation, with MPC based on nonlinear simulation and with open-loop control based on a linear simulation.

model has the following form:

$$
\begin{gathered}
Y_{1}=\sqrt{x^{2}+y^{2}+z^{2}}+\nu_{1}, \\
Y_{2}=\operatorname{atan} 2(x, y)+\nu_{2}, \\
Y_{3}=\operatorname{atan} 2\left(z, \sqrt{x^{2}+y^{2}}\right)+\nu_{3},
\end{gathered}
$$

where $x, y$ and $z$ are the relative $x$-position, relative $y$ position and relative $z$-position, respectively, atan 2 denotes the four quadrant arc-tangent and $\nu_{1}, \nu_{2}$ and $\nu_{3}$ are the measurement noises in each output channel.

We use the Extended Kalman Filter (EKF), based on the CWH equations, to estimate the spacecraft states given the measurements. The output measurement equations are nonlinear and hence the convergence of the EKF estimates is only expected when the state estimate is initialized sufficiently close to the actual state.

\section{Simulation Results}

Three types of maneuvers are considered. The first maneuver is a phasing maneuver with the approaching spacecraft in the same orbit but at a different true anomaly and with the LoS cone oriented along the orbital track. We refer to this as a V-bar docking with an initial spacecraft position along V-bar. The second maneuver is with the initial spacecraft position mostly in the radial direction away from the target and with LoS cone positioned for V-bar docking. We refer to this as V-bar docking with an initial spacecraft position along R-bar. Finally, the third kind of maneuver is with the same initial spacecraft position as in the second maneuver but with the LoS cone positioned for R-bar docking, i.e., oriented along the radial line. In the simulations, the control horizon and the prediction horizon are fixed at 30 steps, with a 2 min update period in the rendezvous phase and 20 sec update period in the docking phase. The switch between rendezvous and docking phases occurs when the estimated spacecraft position enters a $2 \mathrm{~km}$ cube around the target.

\section{A. V-bar docking with initial spacecraft position along V-bar}

The simulated MPC maneuver trajectories for V-bar docking with an initial spacecraft position along V-bar are shown in Figure 3. The MPC controller uses an EKF to estimate the spacecraft states based on range and angle measurements, and it uses the linearized CWH model for prediction. The simulation results are obtained based on the nonlinear relative motion model (1). The EKF estimates converge rapidly to the actual states. Note that the spacecraft motion is three dimensional even though the starting position is in the orbital plane. The out of orbital plane motion is excited since there is a non-zero initial estimation error of the spacecraft position in the z-direction that the MPC controller is trying to correct. Once the docking phase is started, the LoS cone constraint is activated, the trajectory enters into the LoS cone and proceeds towards the origin while satisfying the LoS cone constraint. Large velocity changes are applied initially, which become smaller and more gradual as the spacecraft approaches the origin; control activity increases immediately after entering the docking phase and then decays. The time history of the velocity change direction indicates that the thrust in the direction of the target is minimized as the spacecraft approaches the target.

\section{B. V-bar docking with initial spacecraft position along $R$-bar}

Figure 4 demonstrates a typical maneuver with the LoS cone constraint positioned for a V-bar approach [5]. The spacecraft, with initial position coordinates $[-2,0.126,0]^{\mathrm{T}}$ and initial position coordinate estimates $[-2.8,-0.01,0.1]^{\mathrm{T}}$ starts the motion as in the R-bar approach. Once the docking phase is activated, the trajectory enters into the LoS cone positioned for a V-bar approach and proceeds towards the origin while satisfying the LoS cone constraint. 


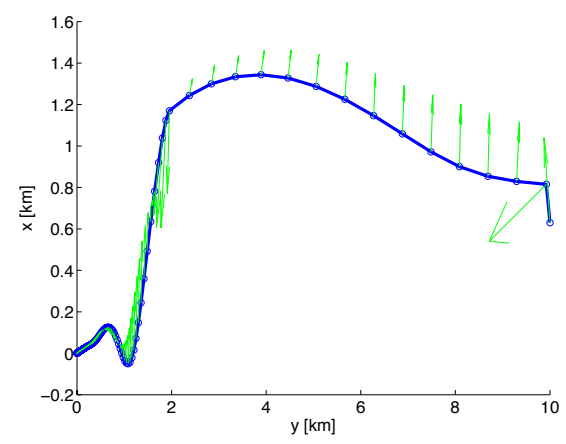

(a)

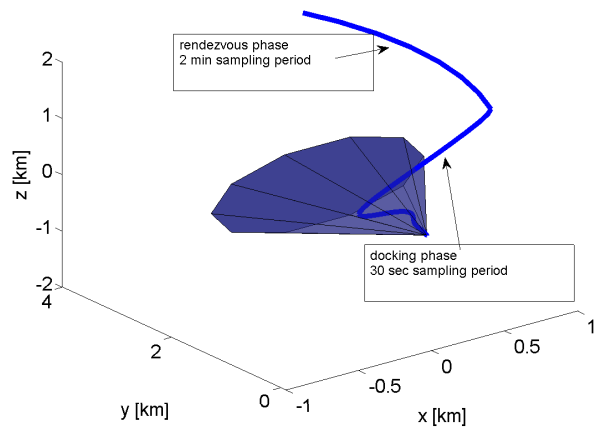

(b)
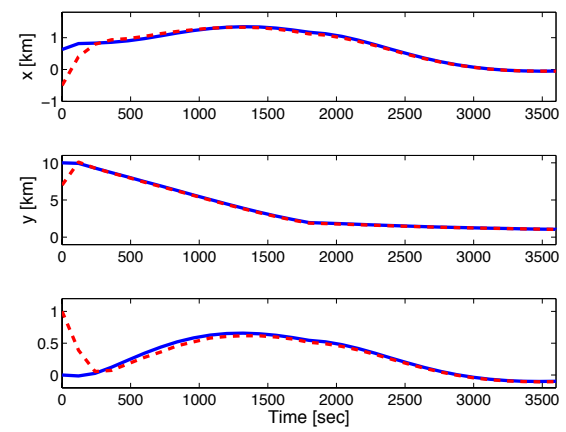

(c)
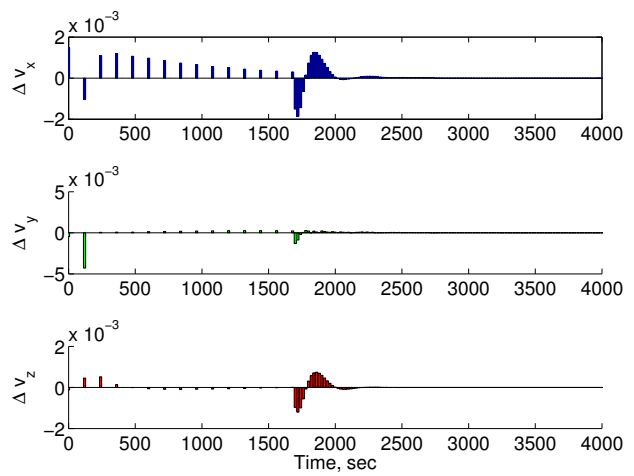

(d)

Fig. 3: (a) Spacecraft trajectory on $x-y$ plane with constraints configured for V-bar docking. (b) Trajectory in 3D with rendezvous and docking phases and LoS cone constraints shown. (c) Spacecraft actual and EKF-estimated relative coordinates (first 30 sample time instants). (d) The three components of $\Delta v$.

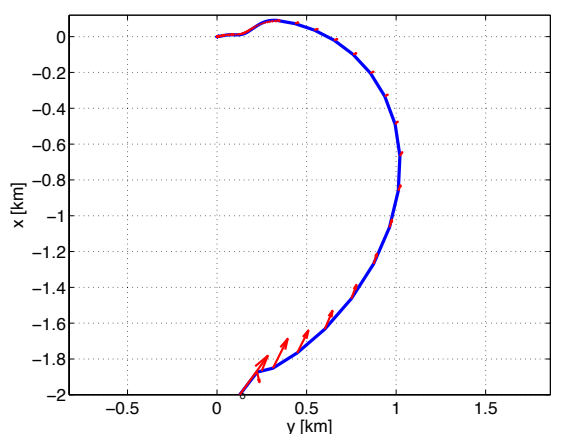

(a)

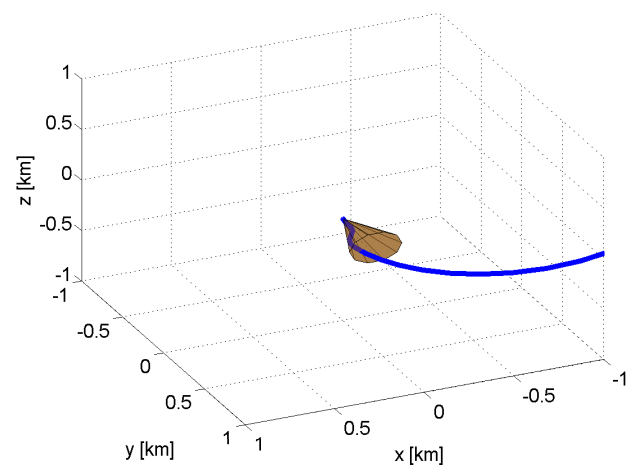

(b)
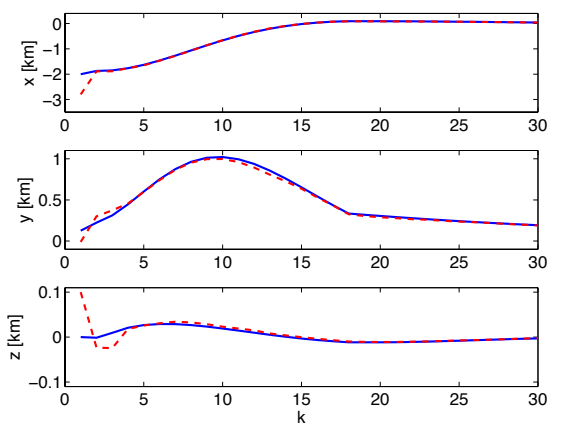

(c)
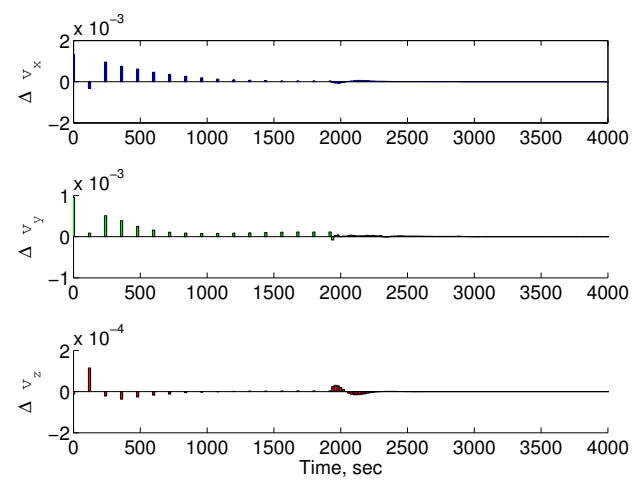

(d)

Fig. 4: (a) Spacecraft trajectory on $x-y$ plane with constraints configured for V-bar docking. (b) Trajectory in 3D with rendezvous and docking phases and LoS cone constraints shown. (c) Spacecraft actual and EKF-estimated relative coordinates (first 30 sample time instants). (d) The three components of $\Delta v$. 
C. $R$-bar docking with initial spacecraft position along $R$ bar

Figure 5 demonstrates a similar maneuver to the one in the previous subsection but for the case when the LoS cone constraint is positioned for an R-bar approach. The behavior is qualitatively similar to the one in Figures 4 . The constraints and maneuver requirements are satisfied using the proposed MPC approach.

\section{CONCLUSION}

In the paper, we demonstrated through simulations on the full nonlinear model that the linear quadratic MPC controller with dynamically reconfigurable constraints can successfully complete three dimensional relative motion maneuvers, when coupled to an EKF, and while relying only on relative angles and relative range measurements.

\section{REFERENCES}

[1] "XSS-11 micro satellite,"

http://www.kirtland.af.mil/shared/media/document/AFD-070404108.pdf

[2] L. Breger, and J. How, Safe trajectories for autonomous rendezvous of spacecraft. Journal of Guidance, Control, and Dynamics, vol. 31, no. $5,2008$.

[3] F. Clark, P. Spehar, J. Brazzel, and H. Hinkel, Laser-based relative navigation and guidance for space shuttle proximity operation. Advances in the Astronautical Sciences, vol. 113, Guidance and Control, pp. 732-737, 2003.

[4] W. Clohessy, and R. Wiltshire, Terminal guidance system for satellite rendezvous. J. Aerospace Sci., vol. 27, no. 9, pp. 653-658, 1960.

[5] W. Fehse. Automated Rendezvous and Docking of Spacecraft. Cambridge Aerospace Series, 2003.

[6] E. Hartley, Model Predictive Control for Spacecraft Rendezvous. Ph.D. Dissertation, University of Cambridge, UK, 2010.

[7] M. Holzinger, J. DiMatteo, J. Schwartz, and M. Milam Passively safe receding horizon control for satellite proximity operations $47 \mathrm{th}$ IEEE Conf. on Decision and Control, pp. 3433-3440, Cancun, Mexico, 2008.

[8] J. Mattingley and S. Boyd. CVXGEN: Code Generation for Convex Optimization. www.cvxgen.com

[9] A. Miele, M. Weeks, and M. Circia, Optimal trajectories for spacecraft rendezvous. Journal of Optimization Theory and Applications, vol. 132,no. 3, pp. 353-376, 2007.

[10] H. Park, S. Di Cairano, and I.V. Kolmanovsky, Model Predictive Control of spacecraft docking with a non-rotating platform, in Proceedings of the 18th IFAC World Congress, 2011.

[11] H. Park, S. Di Cairano, and I.V. Kolmanovsky, "Model Predictive Control for spacecraft rendezvous and docking with a rotating/tumbling platform and for debris avoidance," in Proceedings of American Control Conference, 2011.

[12] H. Park, S. Di Cairano, and I.V. Kolmanovsky, "Linear quadratic Model Predicitve Control approach to spacecraft rendezvous and docking," in Proceedings of 21st AAS/AAIA Space Flight Mechnics Meeting, 2011.

[13] A. Richards, and J. How, Performance evaluation of rendezvous using model predictive control. AIAA Guidance, Navigation, and Control Conference and Exhibit, 2003.

[14] A. Richards, T. Schouwenaars, J. How, and E. Feron, Spacecraft trajectory planning with avoidance constraints using mixed-integer linear programming. Journal of Guidance, Control, and Dynamics, vol. 25, no. 4, pp. 755-764, 2002.

[15] M. Saponara, V. Barrena, A. Bemporad, E.N. Hatley, J. Maciejowski, A. Richards, A. Tramutola, P. Trodden, Model Predictive Control application to spacecraft rendezvous in Mars return scenario, Proceedings of 4th European Conference for Aerospace Sciences (EUCASS), July, 2011, St. Petersburg, Russia.

[16] L. Singh, S. Bortolami, and L. Page, Optimal guidance and thruster control in orbital approach and rendezvous for docking using model predictive control. AIAA Guidance, Navigation, and Control Conference and Exhibit, 2010.

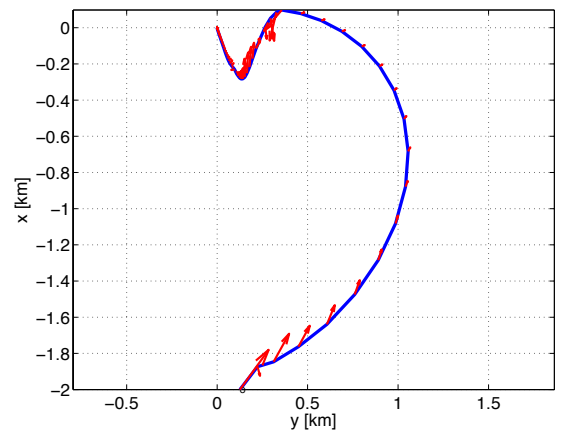

(a)

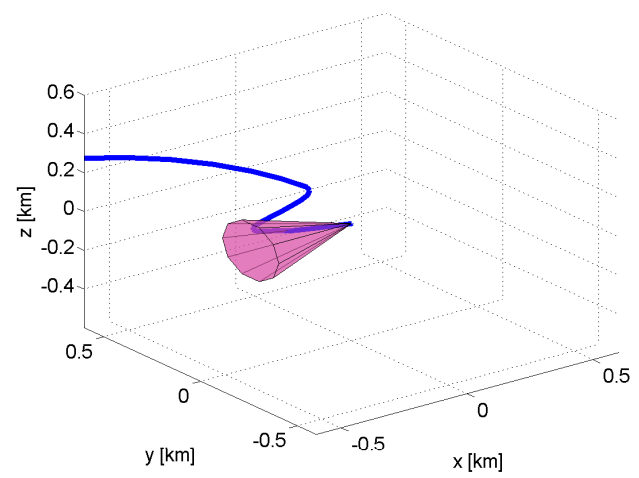

(b)
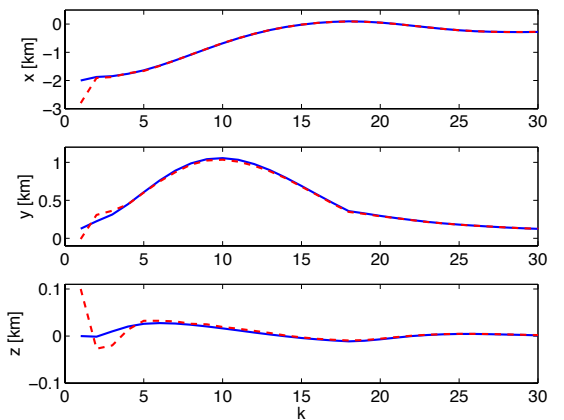

(c)

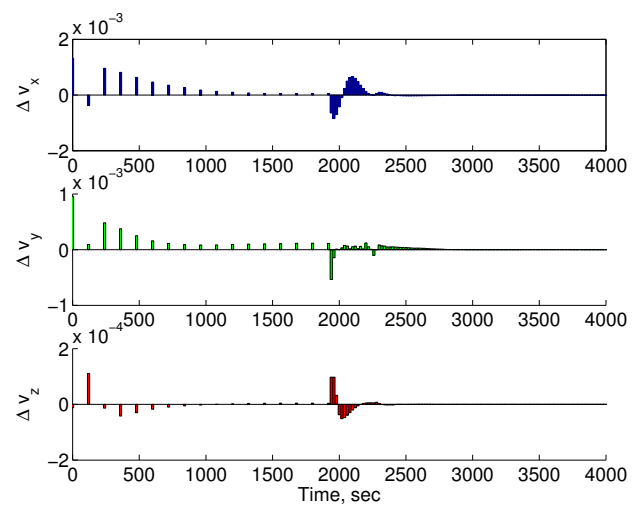

(d)

Fig. 5: (a) Spacecraft trajectory on $x-y$ plane with constraints configured for R-bar docking. (b) Trajectory in 3D with rendezvous and docking phases and LoS cone constraints shown. (c) Spacecraft actual and EKF-estimated relative coordinates. (d) The three components of $\Delta v$. 